\title{
PAIRS OF CARLEMAN-TYPE INTEGRAL EQUATIONS
}

R. C. MAC CAMY ${ }^{1}$

It is known that the singular equation

$$
f(x)=\frac{a(x)}{\pi} \int_{-1}^{+1} f(t)(x-t)^{-1} d t+g(x), \quad|x|<1,
$$

has a solution for all Hölder continuous $a$ and $g$. The integral is the Cauchy principal value. An explicit solution was obtained by Carleman [1]. This note concerns systems of equations of the same type. $f$ and $g$ are then vectors and $a$ is a matrix. We can illustrate the ideas by confining ourselves to two equations and to the case of a constant matrix $a$. Moreover we assume that $g(x)$ is analytic for $x$ in a region $R$ containing the line segment $I_{m} x=0,-1 \leqq x \leqq+1$.

Let $H$ denote the class of vector-valued functions $f=\left(f_{1}, f_{2}\right)$ such that the $f_{i}$ are Hölder continuous on $-1<x<+1$ and satisfy,

$$
f_{i}(x)=O\left((1 \mp x)^{-1+\epsilon}\right) \text { as } x \rightarrow \pm 1
$$

for some $\epsilon>0$.

TheOREM. (i) If $\operatorname{det}(I-i a) \neq 0$ equations (1) possess a solution $f \in H$.

(ii) If $\operatorname{det}(I-i a)=0$ equations (1) possess no solution unless $f \equiv g \equiv 0$.

The existence of a solution in case (i) is a very special case of the general theory of singular integral equations, see for example [2] and [3]. The explicit formula for the solution (equations (9) and (10)) and part (ii) of the theorem appear to be new.

If the matrix $a$ can be diagonalized (1) can be reduced to a system of independent equations but we proceed directly using a vector form of Carleman's method. Suppose $f \in H$ is a (vector) solution of (1). Let $z=x+i y$ and set

$$
F(z)=(2 \pi i)^{-1} \int f(t)(t-z)^{-1} d t
$$

(all integrals are to be understood as from -1 to +1 ). $F(z)$ is analytic on the plane slit along $y=0,|x|<1$. It has limits $F^{+}$and $F^{-}$as $y \rightarrow 0$ from $y>0$ and $y<0$ but these are not the same. The Plemlj formulas yield,

Received by the editors June 8, 1964.

${ }^{1}$ This work was supported by the Air Force Office of Scientific Research. 


$$
\begin{aligned}
& F^{+}-F^{-}=f(x), \\
& F^{+}+F^{-}=-(\pi i)^{-1} \int f(t)(x-t)^{-1} d t,
\end{aligned}
$$

$$
y=0, \quad|x|<1 .
$$

Thus (1) can be rewritten as,

$$
(I+i a) F^{+}=(I-i a) F^{-}+g \text { on } y=0, \quad|x|<1 .
$$

$F(z)$ also satisfies the conditions,

$$
\begin{aligned}
& F(z)=O\left(z^{-1}\right) \text { as } z \rightarrow \infty, \\
& F(z)=O\left((z \mp 1)^{-1+e}\right) \quad \text { as } z \rightarrow \pm 1 .
\end{aligned}
$$

Equations (3) and (4) constitute what is called a Hilbert problem [2]. Case (1). $\operatorname{det}(I-i a) \neq 0$.

Equation (3) can be written as

$$
B F^{+}=F^{-}+G, \quad z=x, \quad|x|<1
$$

where,

$$
B=(I-i a)^{-1}(I+i a), \quad G=(I-i a)^{-1} g .
$$

We introduce the matrices

$$
\psi=(2 \pi i)^{-1} \log B, \quad \phi=[(z-1) /(z+1)]^{\psi} .
$$

These matrices can be defined in terms of the eigenvalues of $B$ and $\psi$. $B$ is the Cayley transform of $a$ hence its eigenvalues $\lambda_{1}$ and $\lambda_{2}$ have the form $\exp \left(i \theta_{1,2}\right), 0 \leqq \theta_{1}, \theta_{2}<2 \pi$. If $\theta_{1} \neq \theta_{2}$ we set

$$
\psi=\left[\left(\theta_{1} \lambda_{2}-\theta_{2} \lambda_{1}\right) I+\left(\theta_{2}-\theta_{1}\right) B\right] / 2 \pi\left(\lambda_{2}-\lambda_{1}\right)
$$

while if $\theta_{1}=\theta_{2}$

$$
\psi=\left[\left(\theta_{1}+i\right) I-i \lambda_{1}^{-1} B\right] / 2 \pi .
$$

The eigenvalues $\tau_{1}$ and $\tau_{2}$ of $\psi$ are $(2 \pi)^{-1} \theta_{1}$ and $(2 \pi)^{-1} \theta_{2}$. We define $\phi$ as

$$
\begin{aligned}
\phi & =\left[\left(\tau_{2} \rho^{\tau_{1}}-\tau_{1} \rho^{\tau_{2}}\right) I+\left(\rho^{\tau_{1}}-\rho^{\tau_{1}}\right) \psi\right] /\left(\tau_{2}-\tau_{1}\right) & & \text { if } \theta_{1} \neq \theta_{2}, \\
& =\left(\rho^{\tau_{1}}-\tau_{1} \rho^{\tau_{1}} \log \rho\right) I+\rho^{\tau_{1}} \log \rho & & \text { if } \theta_{2}=\theta_{1},
\end{aligned}
$$

where $\rho=(z-1) /(z+1)$. Note that the eigenvalues of $\phi$ are $\rho^{\tau_{1}}$ and $\boldsymbol{\rho}^{\boldsymbol{\tau} \boldsymbol{z}} . \phi$ is then a matrix, analytic and nonsingular on the slit plane. It can be verified by the Cayley-Hamilton theorem that

We set

$$
\phi^{-} B=\phi^{+} \text {. }
$$




$$
\chi=\phi F, \quad \mu=\phi^{-} G .
$$

Then if (5) is multiplied by $\phi^{-}$the resulting equation is,

$$
\chi^{+}=\chi^{-}+\mu \text { on } z=x, \quad|x|<1 .
$$

The conditions (4) imply $\left(\tau_{1,2}>0\right)$,

$$
\begin{aligned}
& \chi(z) \rightarrow 0 \text { as } z \rightarrow \infty, \\
& \chi(z)=o\left((z-1)^{-1}\right) \text { as } z \rightarrow 1, \quad \chi(x)=o\left((z+1)^{-2}\right) \text { as } z \rightarrow-1 .
\end{aligned}
$$

The most general function satisfying (7) and (8) is,

$$
\chi(z)=(2 \pi i)^{-1} \int \mu(t)(t-z)^{-1} d t+c(z+1)^{-1}
$$

where $c$ is a constant. $f$ is then determined from (2) as,

$$
f(x)=\left(F^{+}-F^{-}\right)=\left[\left(\phi^{-1}\right)^{+} \chi^{+}-\left(\phi^{-1}\right)^{-} \chi^{-}\right] .
$$

We can prove that (9) and (10) yield a solution of (1) for any choice of the constant $c$. We treat the case $\theta_{1} \neq \theta_{2}$. Define $f$ by (10) and form $F(z)$ as above. Note that $\left(\phi^{ \pm}\right)^{-1}=\left(\phi^{-1}\right)^{ \pm}$so that by (2) and (10) the function

$$
F(z)=\left(\phi^{-1} \chi\right)
$$

is single-valued and vanishes at infinity. It is known that if $\mu(t)$ has algebraic singularities at $t= \pm 1$ the Cauchy integral in (9) possesses singularities of the same orders at $z= \pm 1$. Observe also that $\phi^{-1}$ has the form

$$
A \rho^{-\tau_{1}}+B \rho^{-\tau_{2}}
$$

where $A$ and $B$ are constant matrices. From these facts one can show that $f \in H$ and that $F(z)-\left(\phi^{-1} \chi\right)$ cannot have poles at $z= \pm 1$. Hence

$$
F(z)=\left(\phi^{-1} \chi\right) .
$$

Then we can retrace our steps and establish (3) which is equivalent to (1). This completes the proof for Case (1). The lack of uniqueness is also present for the single equation (see [1]).

Case (2). $\operatorname{det}(I-i a)=0$.

Consider (3) as a system of equations for the components $F_{1}^{+}$and $F_{2}^{+}$. In order to have a solution the right side must be orthogonal to solutions $\nu$ of

$$
\left(I-i a^{T}\right) \nu=0
$$

That is, 


$$
\left((I-i a) F^{-}+g\right) \cdot \nu=F^{-} \cdot\left(I+i a^{T}\right) \nu+g \cdot \nu=\left(2\left(F^{-} \cdot \nu\right)+g \cdot \nu\right)=0 .
$$

Recall that $g(z)$ is analytic in a neighborhood of the slit $y=0,|x|<1$. Thus $2 F \cdot \nu+g \cdot \nu$ is analytic in the slit plane and continuous on $y=0$, $|x|<1$ when defined by its limit value from $y<0$. These limit values are 0 hence $2 F \cdot \nu+g \cdot \nu$ can be continued across $y=0,|x|<1$ as an analytic function and must then be identically zero. In particular

$$
2 F^{+} \cdot \nu+g \cdot \nu=0 \quad \text { on } y=0, \quad|x|<1 .
$$

Now consider (3) as a system of equations for $F^{-}$. We find in the same way that,

$$
2 F^{+} \cdot \tau-g \cdot \tau=0
$$

where $\tau$ is a solution of

$$
\left(I+i a^{T}\right) \tau=0
$$

that is, for $\tau=\bar{\nu}$. Thus

$$
2 F^{+} \cdot \bar{\nu}-g \cdot \bar{\nu}=0 .
$$

Observe that neither $a_{12}$ nor $a_{21}$ can be 0 . Hence we can take $\nu$ in the form $(b, 1)$ with $\operatorname{Im} b \neq 0$. Then (11) and (12) yield,

$$
\begin{aligned}
& 2\left(b F_{1}^{+}+F_{2}^{+}\right)=-b g_{1}-g_{2}, \\
& 2\left(\bar{b} F_{1}^{+}+F_{2}^{+}\right)=+\bar{b} g_{1}+g_{2} .
\end{aligned}
$$

Subtracting we see that $F_{1}^{+}$equals a linear combination of the singlevalued functions $g_{1}$ and $g_{2} . F_{2}^{+}$will then equal a similar combination and thus $F_{1}$ and $F_{2}$ are actually single-valued. Therefore by (2) $f$ will be identically zero.

Our result has application to a boundary-value problem for harmonic functions. Suppose we seek functions $u^{1}$ and $u^{2}$ harmonic in $y>0$, continuous in $y \geqq 0$, becoming at most logarithmically infinite at infinity and satisfying the conditions,

$$
\begin{aligned}
& u_{y}^{i}(x, 0)=0 \text { on }|x|>1, \\
& u_{y}^{i}(x, 0)=\sum_{j=1}^{2} a_{i j} u_{x}^{j}(x, 0)+g^{i}(x), \quad|x|<1 .
\end{aligned}
$$

If a solution exists it can be represented in the form

$$
u^{i}(x, y)=(2 \pi)^{-1} \int f^{i}(t) \log \left[(x-t)^{2}+y^{2}\right] d t .
$$


Then the conditions on $|x|<1$ require that $f^{1,2}$ should be a solution of (1). If $\operatorname{det}(I-i a) \neq 0$ we can find a solution while if $\operatorname{det}(I-i a)=0$ there exists no solution.

\section{REFERENCES}

1. T. Carleman, Sur la résolution de certaines équations intégrales, Mat. Ark. Astronom. Fys. 16, no. 26 (1922).

2. N. I. Muskhelishvili, Singular integral equations, Noordhoff, Groningen, 1953.

3. N. P. Vekua, Systems of singular integral equations and some boundary problems, GITTL, Moscow-Leningrad, 1950.

Carnegie Institute of Technology

\section{A REMARK ON AN ARITHMETIC THEOREM \\ OF CHEVALLEY}

H. BASS

1. Let $k$ be an algebraic number field with ring of integers $\mathcal{O}$, and let $E$ be a finitely generated subgroup of the multiplicative group, $k^{*}$. All but finitely many primes $\mathfrak{p}$ are "prime to $E$," i.e., the units of $\mathcal{O}_{\mathfrak{p}}$ contain $E$. An ideal $\mathfrak{a}$ is called "prime to $E$ " if its prime divisors are. In this case we have a natural homomorphism

$$
E \rightarrow(\mathcal{O} / \mathfrak{a})^{*}
$$

whose kernel, the congruence subgroup $\{a \in E \mid a \equiv 1 \bmod \mathfrak{a}\}$, is evidently of finite index. We denote the group of all (complex) roots of unity by $\boldsymbol{Q} / \boldsymbol{Z}$.

Theorem. Let $\chi: E \rightarrow \boldsymbol{Q} / \boldsymbol{Z}$ be a character of $E$. Then there are infinitely many prime ideals $\mathfrak{p}$ of $k$, prime to $E$, such that $\chi$ factors through a character of $(\Theta / p)^{*}$, i.e., such that $\operatorname{ker}\left(E \rightarrow(\Theta / p)^{*}\right) \subset \operatorname{ker} \chi$.

It follows immediately that if $U$ is a subgroup of finite index in $E$ then $\operatorname{ker}\left(E \rightarrow(\mathcal{O} / \mathfrak{a})^{*}\right) \subset U$ for a suitable $\mathfrak{a}$, which we may take to be square free. This is the form of the theorem proved by Chevalley in [2]. That $\mathfrak{a}$ may be taken square free is implicit in his proof. The following corollary paraphrases Chevalley's theorem.

Corollary 1 (Chevalley). If we embed $E$ in $\prod_{p}$ prime to $E(\theta / p)^{*}$,

Received by the editors August 19, 1964 and, in revised form, September 28, 1964. 